\title{
Photoactive Graphitic Carbon Nitride-Based Gel Beads As Recyclable Photocatalysts
}

\author{
Qian Cao, Jesús Barrio, Markus Antonietti, Baris Kumru,* Menny Shalom,*
}

and Bernhard V. K. J. Schmidt*

Cite This: ACS Appl. Polym. Mater. 2020, 2, 3346-3354

Read Online

ACCESS | Llll Metrics \& More | 国 Article Recommendations ｜ＳＳupporting Information

ABSTRACT: Photocatalysis for clean hydrogen production and wastewater remediation holds great promise for society. However, despite the significant progress in this field, the recyclability of the photocatalytic materials together with good photoactivity remain a great challenge. In here, photocatalytic materials for wastewater cleaning and hydrogen production are based on the utilization of photoactive macrogel beads as the photocatalyst. To do so, we design graphitic carbon nitride-based macrogels with tailored size, swelling behavior, and photocatalytic properties. Detailed studies reveal that the catalytic activity is correlated with the polymer

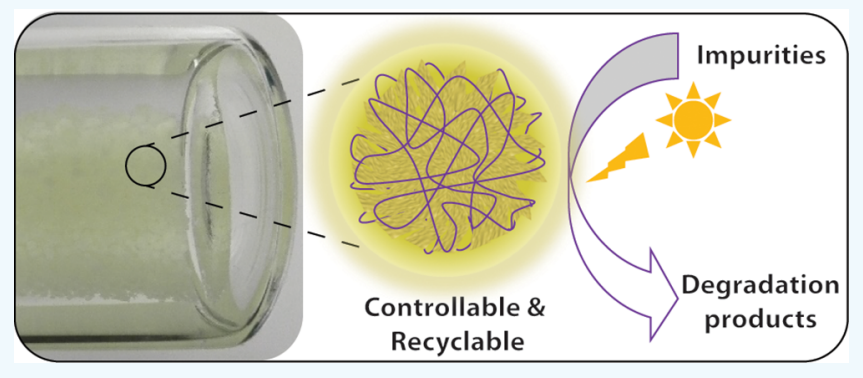
particle size, $\mathrm{g}-\mathrm{C}_{3} \mathrm{~N}_{4}$ content, and swelling behavior, enabling the optimization of the photocatalytic processes. We believe that the presented strategy together with the good photocatalytic activity and excellent recyclability constitute an opening of the path for substantial progress in this field.

KEYWORDS: hydrogel beads, photocatalysis, wastewater remediation, carbon nitride, suspension polymerization, photopolymerization

\section{INTRODUCTION}

Photocatalysis is one of the major strategies to tackle some of the great challenges of our time, including environmental issues such as wastewater remediation ${ }^{1}$ or sustainable energy in hydrogen evolution. ${ }^{2}$ In that regard, graphitic carbon nitride $\left(\mathrm{g}-\mathrm{C}_{3} \mathrm{~N}_{4}\right.$ ) represents a readily accessible photoactive material, which found extensive investigation as a heterogeneous photocatalyst for photoelectrochemisty, ${ }^{3-5}$ water splitting, ${ }^{6}$ or contamination degradation. ${ }^{7}$ Therefore, numerous methods have been developed to improve its photocatalytic performance, e.g., metal or non-metal doping, ${ }^{8}$ surface modification, ${ }^{9}$ morphology and structure modulation, ${ }^{10,11}$ coupling with other materials, ${ }^{12,13}$ and modulation of dispersibility. ${ }^{14,15}$ Among them, the combination of polymers with $\mathrm{g}-\mathrm{C}_{3} \mathrm{~N}_{4}$ results in synergistic effects; e.g., photoactivity can be introduced to polymers through $\mathrm{g}-\mathrm{C}_{3} \mathrm{~N}_{4}$ and facile processing can be introduced to $\mathrm{g}-\mathrm{C}_{3} \mathrm{~N}_{4}$ via polymer incorporation. ${ }^{16}$ For example, our group described the fabrication of polymergrafted $\mathrm{g}-\mathrm{C}_{3} \mathrm{~N}_{4}$ thermoset coatings, which were utilized as photocatalytic surfaces and photoelectrodes. ${ }^{17}$

A combination of photocatalysts with hydrogels and hydrogel particles can provide advances in properties such as recyclability and handling. ${ }^{18,19}$ Of major interest in that regard are gel particles consisting of three-dimensional macromolecular networks that feature high tunability and softness as well as inherent steric stabilization. ${ }^{20,21} \mathrm{Gel}$ particles are used as smart colloidal materials for responding to external stimuli in a fluid environment, e.g., via temperature, ${ }^{22}$ light, ${ }^{23}$ or $\mathrm{pH}^{24}$ Due to the tunability of parameters such as particle size and other properties, these materials have been exploited in a wide range of applications, e.g., drug delivery ${ }^{25}$ or photonic crystals, ${ }^{26}$ and even large-scale applications in industry, such as surface coating, ${ }^{27}$ printing, ${ }^{28}$ and pharmaceutics. ${ }^{29}$ With respect to the size of gel particles, there are nanogels, microgels, and macrogels. Macrogel beads (MGB) are of considerable interest due to easy separation from a fluid medium or to the encapsulation of large entities. ${ }^{30,31}$ There are several methods to fabricate gel particles, namely, through heterophase polymerizations, such as emulsion polymerization, ${ }^{32,33}$ miniemulsion polymerization, ${ }^{34}$ microemulsion polymerization, ${ }^{35}$ inverse suspension polymerization, ${ }^{36}$ or precipitation polymerization. ${ }^{37}$ However, the conventional emulsion polymerization typically focuses on spherical particles with the diameters less than $150 \mathrm{~nm}^{20}$ even with microemulsion polymerizations, the achievable particle sizes are still less than $1 \mu \mathrm{m}$ in diameter. To explore larger MGB, a suspension polymerization must be attempted, in which the polymerization occurs inside of the monomer droplet to obtain solid particles with much larger sizes.

Received: April 28, 2020

Accepted: June 29, 2020

Published: June 29, 2020 
Suspension polymerization is a widely utilized approach for heterophase polymerization in the synthesis of micrometersized particles, such as poly(vinyl chloride) and copolymers, ${ }^{38}$ cross-linked poly(styrene), ${ }^{39}$ poly(methyl methacrylate), ${ }^{40,41}$ and poly(acrylonitrile-butadiene-styrene), ${ }^{42}$ which is welladapted from laboratory to industrial scale. To successfully conduct suspension polymerizations, the dispersed phase should be insoluble in the dispersion medium and stabilized, e.g., oil-in-water (suspension polymerization) or water-in-oil (inverse suspension polymerization). ${ }^{43}$ For hydrophilic monomers, e.g., $N$-isopropylacrylamide, ${ }^{44,45}$ acrylic acid ${ }^{46}$ or $N, N$ dimethylacrylamide (DMA) ${ }^{47}$ inverse suspension polymerization is conducted in nonpolar hydrocarbon media, e.g., paraffin oil, ${ }^{48}$ toluene, ${ }^{49}$ or heptane, ${ }^{36}$ which are needed for dispersing the monomer/water phase. Several studies focus on preparing spherical particles or metal-polymer composites with suspension polymerization. Monodisperse sub-millimetersized microgel particles for human serum albumin removal were described by Takeuchi and co-workers, where they used inverse suspension photopolymerization with various span surfactants under visible light irradiation. ${ }^{43}$

Frequently, radical polymerization techniques are utilized in suspension polymerization, where the initiator is dissolved in the dispersed phase to ensure the entire polymerization takes place within the droplet and solid particles are produced with designed size. In addition, researchers frequently focused on investigating efficient and low energy consuming initiators. ${ }^{50,51}$ In that direction, $\mathrm{g}-\mathrm{C}_{3} \mathrm{~N}_{4}$ was introduced as initiator for polymerization reactions, ${ }^{52}$ as $\mathrm{g}-\mathrm{C}_{3} \mathrm{~N}_{4}$ readily produces radical species under visible light irradiation. ${ }^{53}$ Thus, it can be employed as photoinitiator for radical photopolymerization, ${ }^{54}$ reversible deactivation radical polymerization, ${ }^{55-57}$ or hydrogel formation. ${ }^{58,59}$ While $\mathrm{g}-\mathrm{C}_{3} \mathrm{~N}_{4}$ has been utilized in emulsion

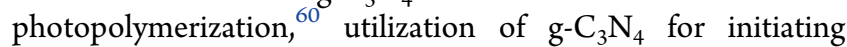
suspension photopolymerization has-to the best of our knowledge- not been reported before. g- $\mathrm{C}_{3} \mathrm{~N}_{4}$ not only acts as a stabilizer for monomer droplet formation ${ }^{61,62}$ and crosslinker ${ }^{60}$ to connect polymer chains during the polymerization but also performs the role of reinforcing agent to enhance the mechanical properties of hydrogels. ${ }^{63,64}$

Introduction of $\mathrm{g}-\mathrm{C}_{3} \mathrm{~N}_{4}$ to inverse suspension polymerization will result beneficially not only for initiating the polymerization process but also for establishing a facile method for fabrication of easily recyclable photocatalytic materials, e.g., for wastewater treatment. In this study, $\mathrm{g}-\mathrm{C}_{3} \mathrm{~N}_{4}$ was utilized as water dispersible photoinitiator to form photocatalytic MGB (Scheme 1). Utilizing cyclohexane as a continuous oil phase and $N, N^{\prime}$-methylenebis(acrylamide) (MBA) as cross-linker,

Scheme 1. Overall Synthetic Procedure for PDMA/g- $\mathrm{C}_{3} \mathrm{~N}_{4}$ MGB via Inverse Suspension Photopolymerization (DMA = $N, N$-Dimethylacrylamide; $\mathrm{MBA}=N, N^{\prime}$ -

Methylenebis(acrylamide); EG = Ethylene Glycol; CMp = Phenyl-Modified $\mathrm{g}-\mathrm{C}_{3} \mathrm{~N}_{4}$ )

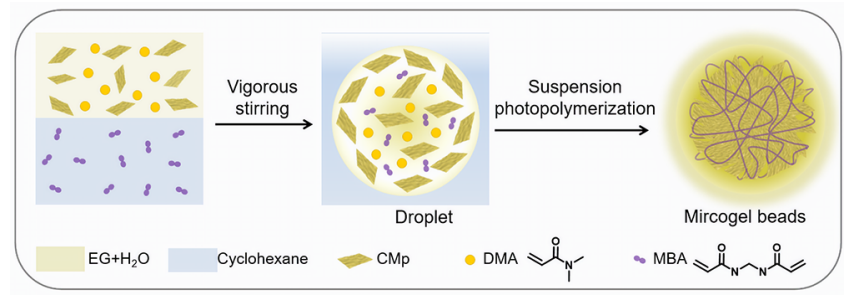

$\mathrm{PDMA} / \mathrm{g}-\mathrm{C}_{3} \mathrm{~N}_{4}$ MGBs with tunable size were synthesized under visible light irradiation. Both cross-linker and $\mathrm{g}-\mathrm{C}_{3} \mathrm{~N}_{4}$ amount influenced the final particle size in the range from 30 $\mu \mathrm{m}$ to $4 \mathrm{~mm}$ with tailored swelling properties. Structure and composition of $\mathrm{PDMA} / \mathrm{g}-\mathrm{C}_{3} \mathrm{~N}_{4}$ MGBs were characterized by scanning electron microscopy (SEM), X-ray diffraction (XRD), and Fourier transform infrared spectroscopy (FTIR). The photophysical properties were investigated by solid state ultraviolet-visible (UV-vis) and photoluminescence (PL) spectroscopy. The photocatalytic activities were evaluated using rhodamine $\mathrm{B}(\mathrm{RhB})$ as a target pollutant and in the hydrogen evolution reaction through water splitting. The novel MGBs show facile size control to modulate photoactivity and indicate distinctive stability and simple recycling.

\section{EXPERIMENTAL SECTION}

Materials. Basic aluminum oxide, cyclohexane (99.5\%), cyanuric acid (98\%), 2,4-diamino-6-phenyl-1,3,5-triazine (97\%), ethylene glycol (EG, 99.5\%), N,N'-methylenebis(acrylamide) (MBA, 99\%), $\mathrm{H}_{2} \mathrm{PtCl}_{6}$ (8\% solution in water), $\mathrm{RhB}(95 \%)$, triethylamine ( $\left.\geq 99.5 \%\right)$, and Tween 20 were purchased from Sigma-Aldrich. N,N-Dimethylacrylamide (DMA, 98\%) was purchased from Sigma-Aldrich and passed through a basic alumina column prior to use to remove the inhibitor. Triethanolamine (TEOA, 99\%) was purchased from J. T. Baker Chemicals. Phenyl-modified $\mathrm{g}-\mathrm{C}_{3} \mathrm{~N}_{4}(\mathrm{CMp})$ was synthesized according to literature. ${ }^{65} 50 \mathrm{~W}$ LED chips (Bridgelux BXRA$50 \mathrm{C} 5300 ; \lambda>410 \mathrm{~nm}$ ) were connected to a self-made circuit and cooling system.

Synthesis of CMp. Phenyl-modified $\mathrm{g}^{-} \mathrm{C}_{3} \mathrm{~N}_{4}$ (CMp) was synthesized according to the literature. ${ }^{65} \mathrm{~A} 1.30 \mathrm{~g}$ amount of cyanuric acid and $1.80 \mathrm{~g}$ of 2,4-diamino-6-phenyl-1,3,5-triazine were weighed and mixed in $50 \mathrm{~mL}$ of deionized water, and then the complex was shaken overnight. Afterward, the precipitate was dried at $60{ }^{\circ} \mathrm{C}$ under vacuum; the mixture was transferred into a crucible, capped, and placed in an oven at $450{ }^{\circ} \mathrm{C}$ for $2 \mathrm{~h}$ under the protection of nitrogen atmosphere, with a heating rate of $2.3{ }^{\circ} \mathrm{C} \mathrm{min}{ }^{-1}$. After cooling to ambient temperature, the yellow CMp powder was collected.

Synthesis of MGB. Different sizes of the MGBs were synthesized from different amounts of CMp, cross-linker, or stirring speed. A certain amount of CMp was dispersed in a mixture of $0.5 \mathrm{~mL}$ of EG and $1 \mathrm{~mL}$ of distilled water; the mixture was ultrasonicated using a $450 \mathrm{D}$ digital sonicator at $50 \%$ amplitude for $15 \mathrm{~min}$ to yield a dispersion. Afterward, $1 \mathrm{~mL}$ of DMA and $10 \mu \mathrm{L}$ of triethylamine were added, named as solution 1 . To a $200 \mathrm{~mL}$ glass vial, $50 \mathrm{~mL}$ of cyclohexane, a certain amount of MBA, and $20 \mu \mathrm{L}$ of Tween 20 were added under vigorous stirring, followed by dropwise addition of solution 1. The vial was capped, and nitrogen gas was flushed for 10 min. The mixture was then put between two $50 \mathrm{~W}$ LED chips to initiate the suspension polymerization. After $12 \mathrm{~h}$ reaction, PDMA/ CMp MGBs with different sizes were formed. The as-prepared particles were washed 3 times with distilled water and freeze-dried to obtain dry MGB samples.

PDMA/CMp-1 to PDMA/CMp-3 were prepared with a CMp amount of $100 \mathrm{mg}$, a stirring speed of $700 \mathrm{rpm}$ and different crosslinker (MBA) amounts of 500, 300, and $150 \mathrm{mg}$, respectively.

PDMA/CMp-4 to PDMA/CMp-6 were prepared with MBA amount of $500 \mathrm{mg}$, a stirrring speed of $700 \mathrm{rpm}$ and different CMp amounts of 30,200 , and $300 \mathrm{mg}$, respectively.

PDMA/CMp-7 and PDMA/CMp- 8 were obtained by the same process as MGB-2 (CMp, $100 \mathrm{mg}, \mathrm{MBA}, 300 \mathrm{mg}$ ) but with stirring speeds of 1200 and $300 \mathrm{rpm}$, respectively.

Photocatalytic RhB Degradation. The photocatalytic activity of the reference $\mathrm{g}-\mathrm{C}_{3} \mathrm{~N}_{4}$ and the PDMA/g- $\mathrm{C}_{3} \mathrm{~N}_{4} \mathrm{MGB}$ samples was evaluated via photocatalytic degradation of $\mathrm{RhB}$ in aqueous solution under visible light irradiation. In a typical $\mathrm{RhB}$ degradation experiment, ${ }^{66} \mathrm{~g}-\mathrm{C}_{3} \mathrm{~N}_{4}$ or a MGB sample $(20 \mathrm{mg})$ and $\mathrm{RhB}$ solution $\left(20 \mathrm{~mL}, 10 \mathrm{mg} \mathrm{mL}^{-1}\right)$ are mixed in a glass vial in the dark under 
continuous stirring for $30 \mathrm{~min}$ in order to achieve an adsorptiondesorption equilibrium. After irradiation with a white $50 \mathrm{~W}$ LED (Bridge lux BXRA-50C5300; $\lambda>410 \mathrm{~nm}$ ) starts, samples are collected after certain time intervals. The RhB concentration was monitored spectrophotometrically utilizing its absorption maximum $(\lambda=554 \mathrm{~nm})$ and plotted as normalized concentration $C / C_{0}$. Additionally, as a reference the same experiment was performed in the absence of photocatalysts.

Photocatalytic $\mathbf{H}_{2}$ Evolution. The hydrogen production was measured as previously reported in a water:TEOA solution (9:1 (v) v)) with $\mathrm{Pt}$ (3 wt \%) as cocatalyst. ${ }^{67}$ The reaction was carried out under argon atmosphere in a Schlenk flask thermally regulated at 25 ${ }^{\circ} \mathrm{C}$ with a cooling system and a white LED array as the irradiation source. In a $50 \mathrm{~mL}$ Schlenk flask, $15 \mathrm{mg}$ of material was suspended in the solvent mixture $(19 \mathrm{~mL})$ composed of water and TEOA in a 9:1 (v/v) ratio and $19.6 \mu \mathrm{L}$ of a $\mathrm{H}_{2} \mathrm{PtCl}_{6}$ solution ( $8 \%$ in water). The mixture underwent constant argon purging during $30 \mathrm{~min}$, and the reaction was started by illuminating with a white $50 \mathrm{~W}$ LED (Bridge lux BXRA-50C5300; $\lambda>410 \mathrm{~nm}$ ). The evolved gas in the headspace of the flask was analyzed by gas chromatography (Agilent 7820 GC system). The apparent quantum yield (AQY) for the $\mathrm{H}_{2}$ evolution reaction was analyzed with an LED (Thorlabs, Model No. M405L4) equipped with a $405 \mathrm{~nm}$ band-pass filter and an average intensity of $35 \mathrm{~mW} \mathrm{~cm}{ }^{-2}$. The measurements were performed in a sealed reactor with continuous argon flow connected to an Agilent 7820 GC system. ${ }^{68}$ After removal of the hydrogen, nitrogen, and oxygen within the reactor cell, the LED irradiation was started and samples were taken every $11 \mathrm{~min}$. The amount of hydrogen was calculated using the integrated area and utilized for the calculation of the AQY as follow: AQY $=N_{\mathrm{e}} / N_{\mathrm{p}} \times 100 \%=2 \mathrm{M} / N_{\mathrm{p}} \times 100 \%$, where $N_{\mathrm{e}}$ are the reaction electrons, $N_{\mathrm{p}}$ the incident photons, and $M$ the amount of $\mathrm{H}_{2}$ molecules.

Characterization. Scanning electron microscopy (SEM) was performed using JSM-7500F (JEOL) equipped with an Oxford Instruments X-MAX $80 \mathrm{~mm}^{2}$ detector for determination of the morphology of MGB. Solid state ultraviolet-visible (UV-vis) spectroscopy of $\mathrm{g}-\mathrm{C}_{3} \mathrm{~N}_{4}$ and MGB were recorded via a Cary 500 Scan spectrophotometer equipped with an integrating sphere, which was used as the device to measure $\mathrm{RhB}$ degradation as well. Photoluminescence of the $\mathrm{g}-\mathrm{C}_{3} \mathrm{~N}_{4}$ and MGB was measured via Jasco spectrofluorometer FP-8300 equipped with a Xe lamp. Fourier transform infrared (FT-IR) spectra were measured on a Nicolet iS 5FT-IR spectrometer. X-ray diffraction (XRD) patterns of MGB was obtained using a Bruker D8 Advance X-ray diffractometer via $\mathrm{Cu} \mathrm{K} \alpha$ radiation. The droplet images were obtained by optical microscopy (OM, DM1000 LED, Leica, Germany).

\section{RESULTS AND DISCUSSION}

Macrogel Synthesis. Size controllable CMp containing PDMA MGB were synthesized via inverse suspension photopolymerization under visible light irradiation. CMp was synthesized according to the literature ${ }^{65}$ and utilized as photoinitiator as it can be well-exfoliated to small particles around $100 \mathrm{~nm}$ via ultrasonication in a water-EG mixture (Supporting Information Figure S1). For the reaction environment, cyclohexane was chosen as the continuous oil phase and MBA as cross-linker as well as a small amount of triethylamine was added to accelerate the radical reaction. ${ }^{52}$ Triethylamine acts as sacrificial electron donor, which enhances photoinitiation efficiency. Water-soluble monomer DMA was polymerized under visible light irradiation, and the specific reaction conditions are collated in Table 1 . The final bead size can be tuned from millimeter to micrometer scale via variation in photoinitiator (CMp) or cross-linker amount during polymerization. As agitation plays an important role for suspension polymerization, the effect of stirring speed was studied as well. To investigate the incorporation of CMp, the
Table 1. Specific Recipe of PDMA/CMp-1 to PDMA/CMp8 and Corresponding Size Distribution and Swelling Ratio $\left(\mathrm{DMA}=1 \mathrm{~mL} ; \mathrm{H}_{2} \mathrm{O}=1 \mathrm{~mL} ; \mathrm{EG}=0.5 \mathrm{~mL}\right.$; Hexane $=50$ $\mathrm{mL}$; Tween $=20 \mu \mathrm{L}$; Triethylamine $=10 \mu \mathrm{L}$ )

\begin{tabular}{cccccc} 
sample name & $\begin{array}{c}\text { CMp } \\
(\mathrm{mg})\end{array}$ & $\begin{array}{c}\text { MBA } \\
(\mathrm{mg})\end{array}$ & $\begin{array}{c}\text { agitation } \\
(\mathrm{rpm})\end{array}$ & \multicolumn{1}{c}{ size $(\mu \mathrm{m})$} & $\begin{array}{c}\text { swelling } \\
\text { ratio }\end{array}$ \\
PDMA/CMp-1 & 100 & 500 & 700 & $1500-2000$ & 1.2 \\
PDMA/CMp-2 & 100 & 300 & 700 & $400-600$ & 1.8 \\
PDMA/CMp-3 & 100 & 125 & 700 & $50-100$ & 2.15 \\
PDMA/CMp-4 & 30 & 500 & 700 & $3000-4000$ & 1.6 \\
PDMA/CMp-5 & 200 & 500 & 700 & $200-300$ & 2.25 \\
PDMA/CMp-6 & 300 & 500 & 700 & $50-150$ & 2.6 \\
PDMA/CMp-7 & 100 & 300 & 1200 & 1000 & 3.5 \\
PDMA/CMp-8 & 100 & 300 & 300 & $30-100$ & 1.1 \\
\hline
\end{tabular}

as-prepared PDMA/CMp MGBs were characterized via XRD (Figure S2). The typical diffraction peak at $27.4^{\circ}$ attributed to 002 interplanar stacking of CMp was observed, which is in good agreement with the literature. ${ }^{65}$ Besides the noncrystalline polymeric network, the stacking peak from XRD is a strong indication of the CMp incorporation. The presence of PDMA in the macrogel can be confirmed via FT-IR (Figure S3). The PDMA/CMp MGB sample features characteristic peaks at $2900 \mathrm{~cm}^{-1}$ and a strong peak at $1700 \mathrm{~cm}^{-1}$ that are assigned to $\mathrm{CH}_{3}$ and $\mathrm{C}=\mathrm{O}$ of PDMA, respectively. The peaks appearing at 1244 and $1580 \mathrm{~cm}^{-1}$ are associated with the typical stretching of $\mathrm{CN}$ heterocycles. Moreover, compared to pure CMp sample, the band at 3014 to $3287 \mathrm{~cm}^{-1}$ can be attributed to hydrogen bonded $\mathrm{N}-\mathrm{H}$ moieties between amine and imine sites shifted after polymerization; this might be due to the interaction between CMp and PDMA polymer chains. Thus, the formation of PDMA/CMp hybrid materials was confirmed. Furthermore, the environment and location of $\mathrm{CMp}$ is indicated that way. Because CMp acts as a stabilizer in the inverse photopolymerization, parts of the material are present at the surface of the MGBs and gel structure, which will be also confirmed via photocatalysis (see below). Moreover, parts of the utilized CMp are in close proximity to PDMA chains.

The most important factor for practical suspension polymerization is the control of the final particle size, which is related to monomer type and initiator amount as well as stirring speed. Herein, in order to investigate the effect of polymerization components on particle size, different amounts of CMp and cross-linker were employed. The size of PDMA/CMp MGB has shown a clear decrease, reaching sizes ranging from $1500-2000 \mu \mathrm{m}$ (Figure 1a), to $400-600 \mu \mathrm{m}$ (Figure $1 \mathrm{~b}$ ) and to $50-100 \mu \mathrm{m}$ (Figure 1c) with decreasing cross-linker amounts of 500, 300, and $125 \mathrm{mg}$, respectively. Digital images (Figure S4) show the size change as well. The cross-linker MBA leads to the formation of a continuous network, which has a considerable effect on overall particle properties. A decreased cross-linker amount results in less cross-linking degree and lower network extent due to the decreased number of joint points, which in turn leads to smaller MGB size and larger pores. As the polymers are formed inside of the water/ monomer droplet, the aggregation of primary polymer networks finally resulted in spherical polymer particle products. Thus, final particle size is affected considerably by the water/ monomer droplet size.

Meanwhile, increasing CMp amount resulted in decreased particles size, with CMp amount increasing from 30 to $300 \mathrm{mg}$, 


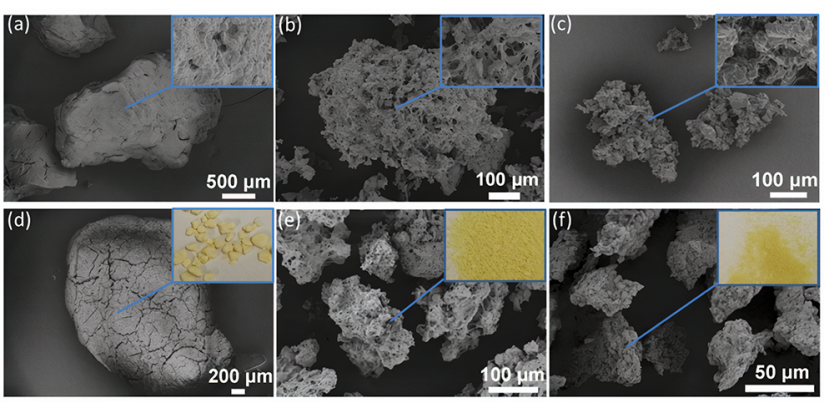

Figure 1. Scanning electron microscopy (SEM) images of PDMA/ CMp-1 (a), PDMA/CMp-2 (b), and PDMA/CMp-3 (c) MGB with decreasing cross-linker amount and PDMA/CMp-4 (d), PDMA/ CMp-5 (e), and PDMA/CMp-6 (f) with increasing CM amount.

the particle size decreased from $3000-4000 \mu \mathrm{m}$ (Figure 1d) to $200-300 \mu \mathrm{m}$ (Figure 1e) and to 50-150 $\mu \mathrm{m}$ (Figure 1f). With defined amounts of DMA and MBA, increased CMp concentration provides more surface area for droplet stabilization; as such more droplets with smaller size were formed. At a given agitation around $700 \mathrm{rpm}$, the droplet sizes were mainly influenced by the balance between droplet breakup and coalescence. Thus, the stabilization effect of $\mathrm{CMp}$ is of considerable importance to the droplet size, which directly affects the final particle size and morphology. For the suspension polymerization, the agitation significantly influenced the particle size as well. The buildup of the final structure inside the monomer droplet depends on the balance of the surrounding stabilizer and the degree of agitation. The monomer droplets tend to be stable in a static condition and result in smaller and denser particles with lower stirring speed (Figure S5). Herein, with the same recipe as PDMA/CMp-2 (700 rpm), PDMA/CMp-7 and PDMA/CMp-8 have shown different particle sizes of $1000 \mu \mathrm{m}$ (Figure S6a) and 20-100 $\mu \mathrm{m}$ (Figure S6b) with different stirring speeds of 1200 and 300 rpm, respectively, which indicated smaller particle formation with lower agitation. As such, the particle size can be tailored via polymerization mixture or polymerization condition.

The swelling properties of the MGB were investigated by immersing the dry material in water (Figure 2) after freezedrying and calculation of the swelling ratios by mass change of swollen and dry MGB. For PDMA/CMp-1 to PDMA/CMp-3

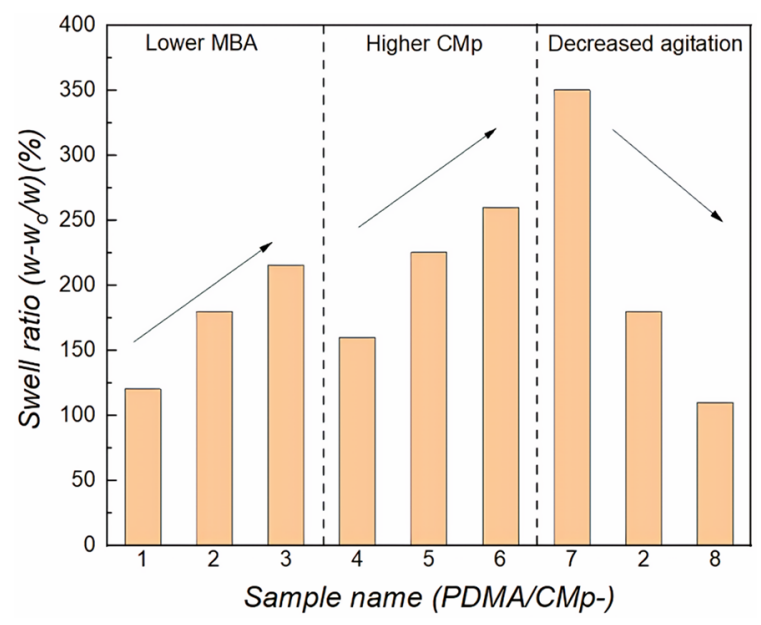

Figure 2. Swelling behavior of PDMA/CMp-1 to PDMA/CMp-8 when immersing in water. samples, the swelling ratios are 1.2 (PDMA/CMp-1), 1.8 (PDMA/CMp-2), and 2.15 (PDMA/CMp-3), which correlate with the decreasing cross-linker content as less covalent crosslinker points lead to less physical restriction for gel particle swelling. When increasing the CMp concentration with constant cross-linker and monomer amount, the MGB showed increased swelling degree in water. In the meantime, smaller particle size was obtained with increased CMp concentration; thus, the increased swelling properties might be a synergetic effect of both size change and CMp content. Then the influence of size on the swelling ratio was investigated as well. With a given cross-linker and CMp content, decreased swelling properties from 3.5-fold (PDMA/CMp-7) to 1.8-fold (PDMA/CMp-2) and to 1.1-fold (PDMA/CMp-8) along with decreased particle size were observed. Overall, the MGB samples show distinctive swelling behavior, which leads to tailored expansion of water absorption and access to CMp. Thus, it is expected that the photocatalytic properties will be correlated with the swelling behavior and ultimately with the synthesis formulation, which was studied in the following.

Optical and Photocatalytic Properties. The optical absorption of the as-prepared PDMA/CMp MGB samples was recorded by UV-vis spectroscopy (Figure 3a); pure CMp shows a relatively wide absorption range from UV to the visible region up to $460 \mathrm{~nm}$, which is ascribed to an appropriate band gap for photocatalysis in the visible light range. ${ }^{65}$ After in situ photopolymerization of PDMA, all of the PDMA/CMp MGB samples exhibited stronger absorption both in $\mathrm{UV}$ and visible light regions compared to pure $\mathrm{CMp}$, which is probably due to increased light scattering from the large MGB. To further investigate the optical properties of the MGB, PL spectroscopy was performed (Figure $3 \mathrm{~b}$ ). Pure CMp possesses a strong peak around $520 \mathrm{~nm}$ with an excitation of $365 \mathrm{~nm}$, which is attributed to the strong recombination of photogenerated electrons and holes with a band gap around $2.7 \mathrm{eV}$. Comparatively, after polymerization, the PL intensity of PDMA/CMp weakens significantly, which might be due to introduction of defects by the addition of PDMA chains. Two separated emission peaks were found (465 and $520 \mathrm{~nm}$ ) for PDMA/CMp MGB. The peak at $520 \mathrm{~nm}$ is attributed to isolated CMp, while the peak at $465 \mathrm{~nm}$ formed due to the attachment of PDMA on CMp. The formation of the additional peak is due to the influence of PDMA grafting on the electron density and band structure of $\mathrm{CMp}$, which indicates the chemical interaction and the cross-linking of CMp with PDMA during the polymerization formed a composite with altered photoluminescent properties. Additionally, this fact confirms the presence of $\mathrm{CMp}$ in the final beads.

RhB Photodegradation. g- $\mathrm{C}_{3} \mathrm{~N}_{4}$ attracted broad interest due to its notable photocatalytic properties and is commonly employed in aqueous systems for pollution degradation. For example, aromatic molecules can be adsorbed on the $\mathrm{g}-\mathrm{C}_{3} \mathrm{~N}_{4}$ surface via $\pi-\pi$ conjugation, which facilitates improved contact for catalytic transformation. However, the powder $\mathrm{g}-\mathrm{C}_{3} \mathrm{~N}_{4}$ has drawbacks such as the separation from the reaction media after photocatalysis, which can be solved with embedding photoactive material into robust networks. ${ }^{69}$ The photocatalytic properties of the MGB were initially studied for degradation of the $\mathrm{RhB}$ dye, which is a colorful additive that is widely used in consumer products, but also causes health and environmental issues. ${ }^{70} \mathrm{RhB}$ is a very suitable test molecule for photodegradation as it is easily detected by spectroscopic means. At 

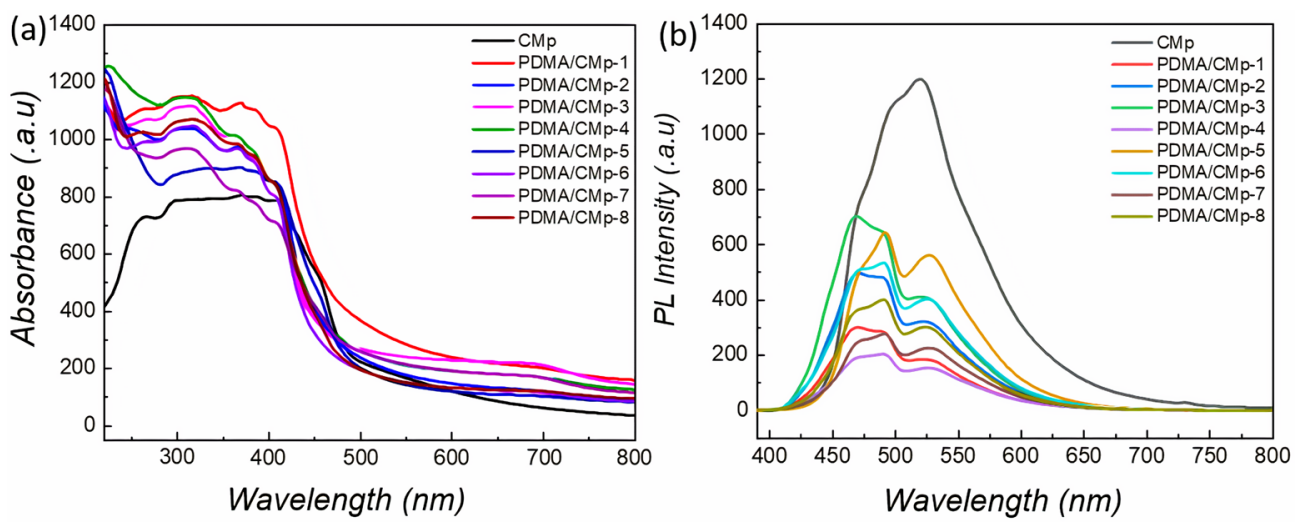

Figure 3. (a) UV-vis absorption spectra of pure CMp and PDMA/CMp MGB and (b) photoluminescence spectra of pure CMp and PDMA/ CMp MGB.

(a)

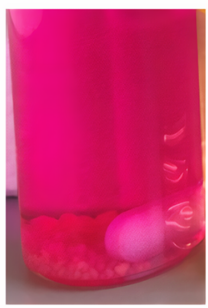

Before

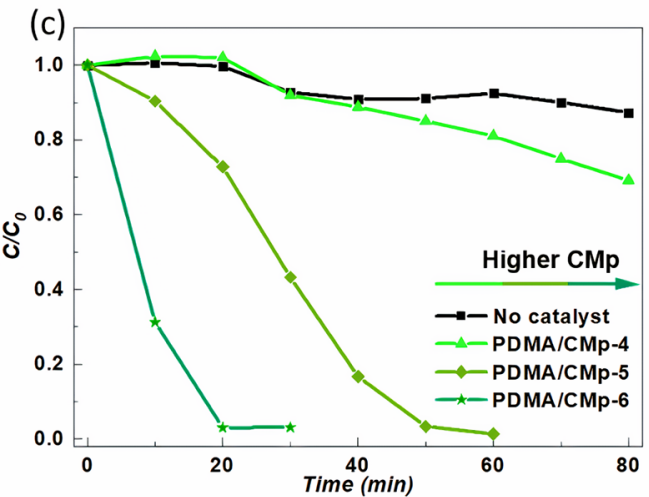

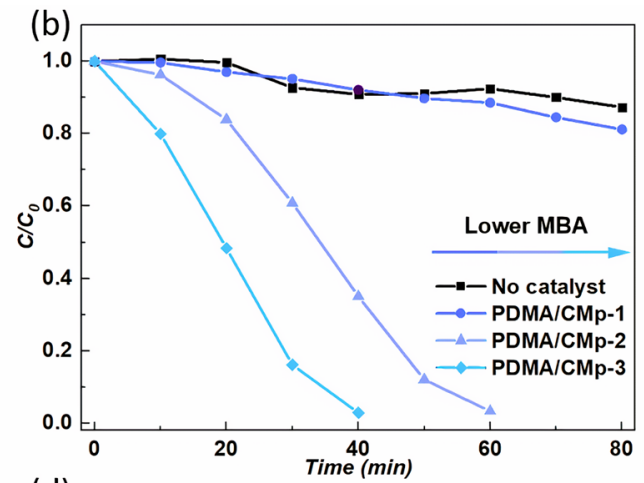

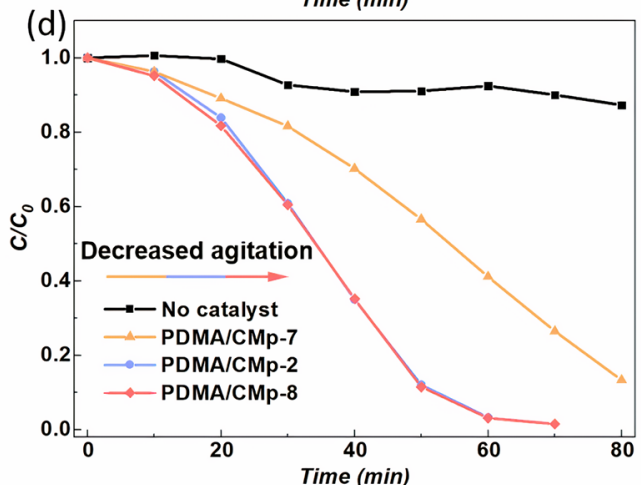

Figure 4. (a) Digital image of RhB solution before and after photodegradation. (b, c, d) show the progress of photocatalytic degradation of RhB in water $\left(10 \mathrm{mg} \mathrm{mL}^{-1}\right)$ under visible light irradiation employing PDMA/CMp MGB samples (after normalization). For comparison, the progress of photocatalytic degradation of $\mathrm{RhB}$ in water $\left(10 \mathrm{mg} \mathrm{mL}^{-1}\right)$ without catalyst is also given.

first, the necessary comparative and control experiments were conducted (Figure S7). Pure RhB solution $\left(10 \mathrm{mg} \mathrm{mL}^{-1}\right)$ with an absorption peak at $554 \mathrm{~nm}$ was degraded under visible light LED illumination in the absence of MGB, which only shows a low degradation extent of $13 \%$ over $80 \mathrm{~min}$, demonstrating that $\mathrm{RhB}$ is quite stable in aqueous solution upon visible light irradiation. However, improved $\mathrm{RhB}$ degradation performance was achieved with the addition of PDMA/CMp MGB into the $\mathrm{RhB}$ solution (Figure 4a). Specifically, in the presence of PDMA/CMp-1 (Figure 4b, Figure S8a), the photodegradation of the dyes slightly increased to $20 \%$ after 80 min light irradiation. Significant improvement of photodegradation performance was observed with the addition of PDMA/ CMp-2 (Figure 4b, Figure S8b) of $97 \%$ after 60 min light irradiation and PDMA/CMp-3 (Figure 4b, Figure S8c) of $97 \%$ after $40 \mathrm{~min}$ light irradiation, respectively. This fact confirms the improvement of the $\mathrm{RhB}$ degradation efficiency with smaller sizes of MGB due to lower cross-linking degree and higher swelling ratio in water, which improves the adsorption of $\mathrm{RhB}$ molecules from solution. Thus, the degradation process is naturally accelerated. Moreover, molecular exchange is improved for materials with higher surface area and porosity. The inset SEM images (Figure $1 \mathrm{a}-\mathrm{c}$, inset) indicate that porosity increases from MGB-1 to MGB-3, which creates more possibilities for contact of beads with dye solution. As such, the contact-based interaction is improved and degradation efficiency is enhanced. Moreover, the PL spectra have shown that the combination with PDMA effectively decreased the radiative recombination, which is also beneficial for the photodegradation activity.

On the other side, the MGB with increased CMp contents exhibited significantly enhanced $\mathrm{RhB}$ photodegradation 

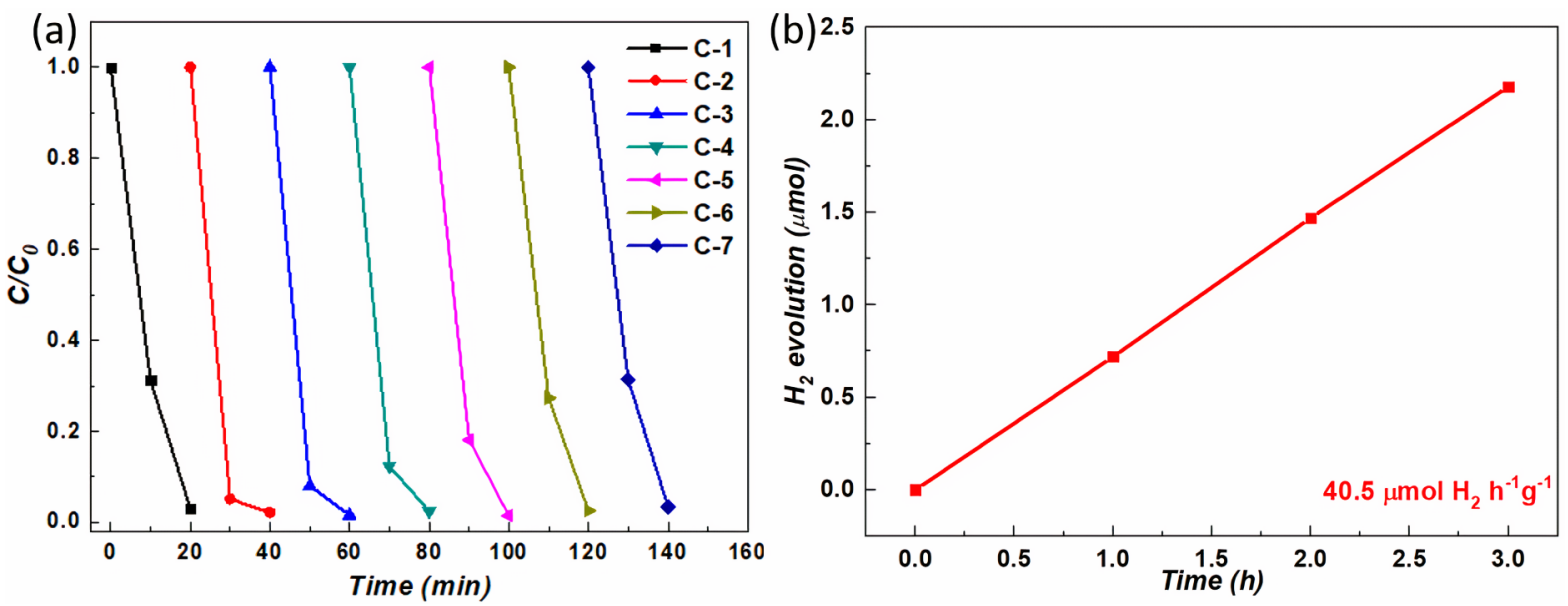

Figure 5. (a) Cyclic photocatalytic degradation of $\mathrm{RhB}$ in several runs (7 times) in the presence of PDMA/CMp-6 MGB under visible light irradiation. (b) Photocatalytic activity of PDMA/CMp-6 for $\mathrm{H}_{2}$ evolution after addition of $\mathrm{Pt}$ cocatalyst carried out in a water/TEOA mixture.

performance; namely, PDMA/CMp-4 reached 31\% after 80 min irradiation and PDMA/CMp-5 97\% after $50 \mathrm{~min}$, respectively (Figure 4c, Figure S9). When the CMp amount in the MGB formation reaction increased to $300 \mathrm{mg}$, PDMA/ CMp-6 exhibited superior photodegradation behavior of $97 \%$ only after $20 \mathrm{~min}$, which is attributed to the fact that CMp is the only component present in MGB that is responsible for the photocatalytic degradation. Therefore, increasing the CMp content will contribute to enhanced photoactive species; consequently a faster photodegradation process is expected upon raising the $\mathrm{CMp}$ content. At the same time, modifying the bead size while increasing CMp content may also play a significant role in the $\mathrm{RhB}$ photodegradation, which will be discussed later on. Nevertheless, the reason for the high photoactivity of PDMA/CMp-6 is the synergistic effect between the catalytically active sites and the small bead size. In general, the connection of CMp and PDMA to form internal cross-linked structure influenced the band structure of CMp and decreased the number of active species. However, the tunability of particle sizes by modulating the polymerization component while maintaining the photoactivity in different scenarios is a significant feature of the presented MGBs.

The influence of different factors (e.g., size, CMp, or crosslinker content) in the photodegradation efficiency was further studied. The photodegradation activities of PDMA/CMp-7 and PDMA/CMp- 8 were conducted under the same condition as previous experiments (Figure 4d, Figure S10). Interestingly, PDMA/CMp-7 leads to a photodegradation conversion of $87 \%$ after 80 min irradiation, while PDMA/CMp- 8 almost possesses the same degradation property as PDMA/CMp-2 of $97 \%$ after $60 \mathrm{~min}$ irradiation. Similar to the results observed previously, the largest bead size features the lowest activity despite the large extent of swelling of PDMA/CMp-7 (3.5 fold), while the intermediate and smallest size display a similar photocatalytic performance. These phenomena indicate that there is an optimum in the system between different factors, which can be mainly correlated to the bead size. As the magnified SEMs indicate, the smaller particle size possesses more porous structures (Figure 1a-c inset, Figure S11). As such, smaller beads provide more opportunities for interfacial contact with dye solution and contribute to enhanced photoreaction activity. Overall, the MGB polymerized with lower cross-linker amount and higher CMp amount, as long as particles were formed successfully, led to the most efficient photodegradation activity. Additionally, the $\mathrm{RhB}$ degradation performance of CMp was measured as a reference (Figure S12). A very similar performance to that of PDMA/CMp-6 was observed, which confirms a minor influence of the cross-linked structure on the overall photocatalytic performance of the MGBs. It should be noted that the intensity of the $\mathrm{RhB}$ related absorption peak approaches zero in most cases during photodegradation. Nevertheless, the formation of an absorption peak at lower wavelengths is observed, which could be due to side product formation and is also responsible for slight coloration of the solution after degradation (Figure 4a).

In addition to facile size control to modulate different scenarios, another advantage for the MGB compared to powder materials is the excellent swelling properties as well as facile processing and recycling after photodegradation activities, which is also an important factor for application as photocatalyst. Thus, PDMA/CMp-6 MGB was further utilized for recycling tests (Figure 5a); $20 \mathrm{mg}$ of MGB were introduced into $20 \mathrm{~mL}$ of $\mathrm{RhB}\left(10 \mathrm{mg} \mathrm{mL} \mathrm{mL}^{-1}\right)$ solution. The $\mathrm{RhB}$ degradation rate exhibited a slight decrease after irradiation for 60 min only (Figure S13), as it still demonstrated a 97\% extent of $\mathrm{RhB}$ degradation within $20 \mathrm{~min}$ after 7 times recycling, suggesting that the MGBs possess sufficient stability and recyclability for $\mathrm{RhB}$ degradation, which is of considerable importance for its potential application in wastewater remediation.

Hydrogen Evolution. In order to further analyze the photocatalytic activity of the prepared MGBs, the hydrogen evolution reaction through water splitting was carried out in a water/triethanolamine (TEOA) mixture with $\mathrm{Pt}$ as cocatalyst and the same irradiation source as in the case of the RhB experiment. The most photoactive sample for the RhB degradation, PDMA/CMp-6, produced hydrogen at a rate of $40.5 \mu \mathrm{mol}$ of $\mathrm{H}_{2} \mathrm{~h}^{-1} \mathrm{~g}^{-1}$ (Figure $5 \mathrm{~b}$ ), which corresponds to an average quantum yield (AQY) of around $1.0 \%$ upon illumination at $405 \mathrm{~nm}$. The AQY remained stable up to more than $12 \mathrm{~h}$ despite phenomena such as evaporation of the reaction media within the reactor (Figure S14). Despite higher values reported in the literature, we want to note that the intrinsic $\mathrm{g}_{-} \mathrm{C}_{3} \mathrm{~N}_{4}$ photoactivity for the hydrogen evolution reaction was preserved in the prepared MGBs after altering its optical and electronic properties through polymerization. 
Therefore, achieving an optimum between precise tailoring of particle size and chemical composition and photoactivity. As such, the presented approach opens up new opportunities for tailored photocatalytic materials.

\section{CONCLUSION}

Polymeric materials with photocatalytic properties have a significant impact on current technology, e.g., for wastewater remediation or hydrogen evolution. In here, PDMA/CMp MGBs were successfully synthesized via inverse suspension photopolymerization employing $\mathrm{CMp}$ as photoinitiator. The structure and morphology could be controlled and adjusted by modulating agitation as well as CMp or cross-linker content during polymerization, which had considerable effect on swelling behavior, i.e., a swelling ratio between 1.1- and 3.5fold, depending on composition. The optical properties were investigated showing extended light absorption properties compared to pure $\mathrm{CMp}$ due to inner interaction between PDMA and CMp. Both, excellent swelling behavior in water and quenched PL intensity benefit the photocatalytic properties. The photocatalytic activities were evaluated via photodegradation of $\mathrm{RhB}$ under visible light irradiation, and the hydrogen evolution reaction with considerable results. The advantage of PDMA/CMp MGBs for photodegradation and hydrogen evolution reaction is the tunability as well as stability and recyclability. The present PDMA/CMp MGBs provide a new strategy to gel particle engineering, as $\mathrm{g}^{-} \mathrm{C}_{3} \mathrm{~N}_{4}$ containing gel particles can be fabricated with high precision and tailored toward the needs, which might demonstrate potential gel particle materials for photocatalytic applications, maybe even on an industrial scale.

\section{ASSOCIATED CONTENT}

\section{S1 Supporting Information}

The Supporting Information is available free of charge at https://pubs.acs.org/doi/10.1021/acsapm.0c00453.

Additional synthetic procedures; digital photographs; SEM, XRD, FT-IR, and UV-vis spectra; and AQY measurements (PDF)

\section{AUTHOR INFORMATION}

\section{Corresponding Authors}

Baris Kumru - Department of Colloid Chemistry, Max Planck Institute of Colloids and Interfaces, 14476 Potsdam, Germany; Email: baris.kumru@mpikg.mpg.de

Menny Shalom - Department of Chemistry and Ilse Katz Institute for Nanoscale Science and Technology, Ben-Gurion University of the Negev, Ben-Beer-Sheva 8410501, Israel; ○ orcid.org/0000-0002-4506-4177; Email: mennysh@ bgu.ac.il

Bernhard V. K. J. Schmidt - Department of Colloid Chemistry, Max Planck Institute of Colloids and Interfaces, 14476 Potsdam, Germany; School of Chemistry, University of Glasgow,

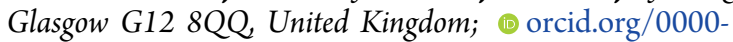
0002-3580-7053; Email: bernhard.schmidt@glasgow.ac.uk

\section{Authors}

Qian Cao - Department of Colloid Chemistry, Max Planck Institute of Colloids and Interfaces, 14476 Potsdam, Germany Jesús Barrio - Department of Chemistry and Ilse Katz Institute for Nanoscale Science and Technology, Ben-Gurion University of the Negev, Ben-Beer-Sheva 8410501, Israel; 이이.org/ 0000-0002-4147-2667

Markus Antonietti - Department of Colloid Chemistry, Max Planck Institute of Colloids and Interfaces, 14476 Potsdam,

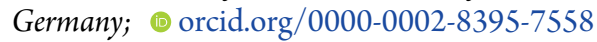

Complete contact information is available at:

https://pubs.acs.org/10.1021/acsapm.0c00453

\section{Notes}

The authors declare no competing financial interest.

\section{ACKNOWLEDGMENTS}

We thank the Max Planck Society for financial support and funding of open access. B.S. acknowledges the University of Glasgow for support. M.S. and J.B. thank the Minerva Center No. 117873 for support. We thank Ms. Junyi Li for fruitful discussion and help with materials characterization.

\section{REFERENCES}

(1) Chakrabarti, S.; Dutta, B. K. Photocatalytic degradation of model textile dyes in wastewater using $\mathrm{ZnO}$ as semiconductor catalyst. J. Hazard. Mater. 2004, 112 (3), 269-278.

(2) Maeda, K.; Teramura, K.; Lu, D.; Takata, T.; Saito, N.; Inoue, Y.; Domen, K. Photocatalyst releasing hydrogen from water. Nature 2006, 440 (7082), 295-295.

(3) Zhang, G.; Li, G.; Lan, Z. A.; Lin, L.; Savateev, A.; Heil, T.; Zafeiratos, S.; Wang, X.; Antonietti, M. Optimizing optical absorption, exciton dissociation, and charge transfer of a polymeric carbon nitride with ultrahigh solar hydrogen production activity. Angew. Chem., Int. Ed. 2017, 56 (43), 13445-13449.

(4) Volokh, M.; Peng, G.; Barrio, J.; Shalom, M. Carbon nitride materials for water splitting photoelectrochemical cells. Angew. Chem., Int. Ed. 2019, 58 (19), 6138-6151.

(5) Liu, C.; Chen, Z.; Wang, Z.; Li, W.; Ju, E.; Yan, Z.; Liu, Z.; Ren, J.; $\mathrm{Qu}, \mathrm{X}$. A graphitic hollow carbon nitride nanosphere as a novel photochemical internalization agent for targeted and stimuliresponsive cancer therapy. Nanoscale 2016, 8 (25), 12570-12578.

(6) Oh, J.; Lee, J. M.; Yoo, Y.; Kim, J.; Hwang, S.-J.; Park, S. New insight of the photocatalytic behaviors of graphitic carbon nitrides for hydrogen evolution and their associations with grain size, porosity, and photophysical properties. Appl. Catal., B 2017, 218, 349-358.

(7) Peng, G.; Qin, J.; Volokh, M.; Shalom, M. Freestanding hierarchical carbon nitride/carbon-paper electrode as a photoelectrocatalyst for water splitting and dye degradation. ACS Appl. Mater. Interfaces 2019, 11 (32), 29139-29146.

(8) Jiang, L.; Yuan, X.; Pan, Y.; Liang, J.; Zeng, G.; Wu, Z.; Wang, H. Doping of graphitic carbon nitride for photocatalysis: a reveiw. Appl. Catal., B 2017, 217, 388-406.

(9) Cruz, D.; Garcia Cerrillo, J.; Kumru, B.; Li, N.; Dario Perea, J.; Schmidt, B. V. K. J.; Lauermann, I.; Brabec, C. J.; Antonietti, M. Influence of thiazole-modified carbon nitride nanosheets with feasible electronic properties on inverted perovskite solar cells. J. Am. Chem. Soc. 2019, 141 (31), 12322-12328.

(10) Yang, Z.; Zhang, Y.; Schnepp, Z. Soft and hard templating of graphitic carbon nitride. J. Mater. Chem. A 2015, 3 (27), 1408114092.

(11) Barrio, J.; Shalom, M. Rational Design of Carbon Nitride Materials by Supramolecular Preorganization of Monomers. ChemCatChem 2018, 10 (24), 5573-5586.

(12) Ong, W.-J. 2D/2D graphitic carbon nitride (g-C3N4) heterojunction nanocomposites for photocatalysis: why does face-toface interface matter? Front. Mater. 2017, 4, 11.

(13) Fu, J.; Yu, J.; Jiang, C.; Cheng, B. g-C3N4-Based Heterostructured Photocatalysts. Adv. Energy Mater. 2018, 8 (3), 1701503.

(14) Kim, J. K.; Park, S.; Yoo, R. J.; Jeong, H. J.; Oh, J.; Lee, Y. J.; Park, S.; Kim, D. W. Thin PEGylated carbon nitrides: water- 
dispersible organic nanodots as bioimaging probes. Chem. - Eur. J. 2018, 24 (14), 3506-3511.

(15) Kumru, B.; Antonietti, M.; Schmidt, B. V. K. J. Enhanced Dispersibility of Graphitic Carbon Nitride Particles in Aqueous and Organic Media via a One-Pot Grafting Approach. Langmuir 2017, 33 (38), 9897-9906.

(16) Cao, Q.; Kumru, B.; Antonietti, M.; Schmidt, B. V. K. J. Graphitic carbon nitride and polymers: a mutual combination for advanced properties. Mater. Horiz. 2020, 7 (3), 762-786.

(17) Kumru, B.; Barrio, J.; Zhang, J.; Antonietti, M.; Shalom, M.; Schmidt, B. V. K. J. Robust carbon nitride-based thermoset coatings for surface modification and photochemistry. ACS Appl. Mater. Interfaces 2019, 11 (9), 9462-9469.

(18) Ferguson, C. T.; Huber, N.; Landfester, K.; Zhang, K. A. Dualresponsive photocatalytic polymer nanogels. Angew. Chem. 2019, 131 (31), 10677-10681.

(19) Ye, B.; Yao, C.; Yan, M.; Zhang, H.; Xi, F.; Liu, J.; Li, B.; Dong, $X$. Photo-Induced Hydrogel Formation Based on g-C3N4 Nanosheets with Self-Cross-Linked 3D Framework for UV Protection Application. Macromol. Mater. Eng. 2019, 304, 1800500.

(20) Saunders, B. R.; Vincent, B. Microgel particles as model colloids: theory, properties and applications. Adv. Colloid Interface Sci. 1999, 80 (1), 1-25.

(21) Plamper, F. A.; Richtering, W. Functional microgels and microgel systems. Acc. Chem. Res. 2017, 50 (2), 131-140.

(22) Ivashkov, O. V.; Yakimova, T. M.; Evtushenko, E. G.; Gelissen, A. P.; Plamper, F. A.; Richtering, W.; Yaroslavov, A. A. On the mechanism of payload release from liposomes bound to temperaturesensitive microgel particles. Colloids Surf., A 2019, 570, 396-402.

(23) Lehmann, M.; Tabaka, W.; Möller, T.; Oppermann, A.; Wöll, D.; Volodkin, D.; Wellert, S.; Klitzing, R. v. Dls setup for in situ measurements of photoinduced size changes of microgel-based hybrid particles. Langmuir 2018, 34 (12), 3597-3603.

(24) Pieprzyk, S.; Heyes, D.; Brańka, A. Spatially dependent diffusion coefficient as a model for $\mathrm{pH}$ sensitive microgel particles in microchannels. Biomicrofluidics 2016, 10 (5), 054118.

(25) Muratalin, M.; Luckham, P. F.; Esimova, A.; Aidarova, S.; Mutaliyeva, B.; Madybekova, G.; Sharipova, A.; Issayeva, A. Study of n-isopropylacrylamide-based microgel particles as a potential drug delivery agents. Colloids Surf., A 2017, 532, 8-17.

(26) Park, J.-G.; Rogers, W. B.; Magkiriadou, S.; Kodger, T.; Kim, S.H.; Kim, Y.-S.; Manoharan, V. N. Photonic-crystal hydrogels with a rapidly tunable stop band and high reflectivity across the visible. Opt. Mater. Express 2017, 7 (1), 253-263.

(27) Bridges, A. W.; Singh, N.; Burns, K. L.; Babensee, J. E.; Lyon, L. A.; Garcia, A. J. Reduced acute inflammatory responses to microgel conformal coatings. Biomaterials 2008, 29 (35), 4605-4615.

(28) Peng, J.; Zhao, D.; Tang, X.; Tong, F.; Guan, L.; Wang, Y.; Zhang, M.; Cao, T. Cool microcontact printing to fabricate thermosensitive microgel patterns. Langmuir 2013, 29 (38), 1180911814.

(29) Eral, H. B.; Lopez-Mejias, V.; O’Mahony, M.; Trout, B. L.; Myerson, A. S.; Doyle, P. S. Biocompatible alginate microgel particles as heteronucleants and encapsulating vehicles for hydrophilic and hydrophobic drugs. Cryst. Growth Des. 2014, 14 (4), 2073-2082.

(30) Gotoh, T.; Matsushima, K.; Kikuchi, K.-I. Preparation of alginate-chitosan hybrid gel beads and adsorption of divalent metal ions. Chemosphere 2004, 55 (1), 135-140.

(31) Tan, W. H.; Takeuchi, S. Monodisperse alginate hydrogel microbeads for cell encapsulation. Adv. Mater. 2007, 19 (18), 26962701.

(32) Etchenausia, L.; Deniau, E.; Brûlet, A.; Forcada, J.; Save, M. Cationic thermoresponsive poly (N-vinylcaprolactam) microgels synthesized by emulsion polymerization using a reactive cationic macro-RAFT agent. Macromolecules 2018, 51 (7), 2551-2563.

(33) Tuncer, C.; Samav, Y.; Ülker, D.; Baker, S. B.; Bütün, V. Multiresponsive microgel of a water-soluble monomer via emulsion polymerization. J. Appl. Polym. Sci. 2015, 132 (24), 42072.
(34) Lu, F.; Luo, Y.; Li, B.; Zhao, Q.; Schork, F. J. Synthesis of thermo-sensitive nanocapsules via inverse miniemulsion polymerization using a PEO-RAFT agent. Macromolecules 2010, 43 (1), 568571.

(35) Neyret, S.; Vincent, B. The properties of polyampholyte microgel particles prepared by microemulsion polymerization. Polymer 1997, 38 (25), 6129-6134.

(36) Dowding, P. J.; Vincent, B.; Williams, E. Preparation and swelling properties of poly(NIPAM) "Minigel" particles prepared by inverse suspension polymerization. J. Colloid Interface Sci. 2000, 221 (2), 268-272.

(37) Hu, X.; Tong, Z.; Lyon, L. A. Control of poly (Nisopropylacrylamide) microgel network structure by precipitation polymerization near the lower critical solution temperature. Langmuir 2011, 27 (7), 4142-4148.

(38) Bao, Y. Z.; Huang, Z. M.; Weng, Z. X. Preparation and characterization of poly (vinyl chloride)/layered double hydroxides nanocomposite via in situ suspension polymerization. J. Appl. Polym. Sci. 2006, 102 (2), 1471-1477.

(39) Kumru, B.; Gure, B.; Bicak, N. Regio-selective peroxybromination of poly (vinyl methyl ketone) as versatile tool for generation active ATRP initiation sites on solid surfaces. J. Polym. Sci., Part A: Polym. Chem. 2013, 51 (18), 3892-3900.

(40) Wang, J.; Hu, H.; Wang, X.; Xu, C.; Zhang, M.; Shang, X. Preparation and mechanical and electrical properties of graphene nanosheets-poly (methyl methacrylate) nanocomposites via in situ suspension polymerization. J. Appl. Polym. Sci. 2011, 122 (3), 18661871.

(41) Kim, J.-W.; Shim, J.-W.; Bae, J.-H.; Han, S.-H.; Kim, H.-K.; Chang, I.-S.; Kang, H.-H.; Suh, K.-D. Titanium dioxide/poly (methyl methacrylate) composite microspheres prepared by in situ suspension polymerization and their ability to protect against UV rays. Colloid Polym. Sci. 2002, 280 (6), 584-588.

(42) Rodriguez-Tobias, H.; Morales, G.; Rodriguez-Fernandez, O.; Acuna, P. Mechanical and UV-shielding properties of in situ synthesized poly (acrylonitrile-butadiene-styrene)/zinc oxide nanocomposites. J. Appl. Polym. Sci. 2013, 127 (6), 4708-4718.

(43) Takimoto, K.; Takano, E.; Kitayama, Y.; Takeuchi, T. Synthesis of monodispersed submillimeter-sized molecularly imprinted particles selective for human serum albumin using inverse suspension polymerization in water-in-oil emulsion prepared using microfluidics. Langmuir 2015, 31 (17), 4981-7.

(44) Duan, L.; Chen, M.; Zhou, S.; Wu, L. Synthesis and characterization of poly ( $N$-isopropylacrylamide)/silica composite microspheres via inverse Pickering suspension polymerization. Langmuir 2009, 25 (6), 3467-3472.

(45) O'connor, P.; Zetterlund, P. B.; Aldabbagh, F. Nitroxidemediated stabilizer-free inverse suspension polymerization of $\mathrm{N}$ isopropylacrylamide in supercritical carbon dioxide. J. Polym. Sci., Part A: Polym. Chem. 2011, 49 (7), 1719-1723.

(46) Klinpituksa, P.; Kosaiyakanon, P. Superabsorbent polymer based on sodium carboxymethyl cellulose grafted polyacrylic acid by inverse suspension polymerization. Int. J. Polym. Sci. 2017, 2017, 3476921.

(47) Panahi, H. A.; Mehramizi, A.; Hosseinifard, Z.; Moniri, E. Synthesis and characterization of new molecular imprinting poly [1( $N, \quad N$-bis-carboxymethyl) amino-3-allylglycerol-co-dimethylacrylamide] for selective sorption and determination of cefuroxime sodium in biological and pharmaceutical samples. React. Funct. Polym. 2013, 73 (1), 132-140.

(48) Xie, G.; Pan, P.; Bao, Y. Morphology and blowing agent encapsulation efficiency of vinylidene chloride copolymer microspheres synthesized by suspension polymerization in the presence of a blowing agent. J. Appl. Polym. Sci. 2017, 134 (4), 44376.

(49) Omidian, H.; Zohuriaan-Mehr, M. J.; Bouhendi, H. Polymerization of sodium acrylate in inverse-suspension stabilized by sorbitan fatty esters. Eur. Polym. J. 2003, 39 (5), 1013-1018. 
(50) Shao, J.; Huang, Y.; Fan, Q. Visible light initiating systems for photopolymerization: status, development and challenges. Polym. Chem. 2014, 5 (14), 4195-4210.

(51) Yagci, Y.; Jockusch, S.; Turro, N. J. Photoinitiated polymerization: advances, challenges, and opportunities. Macromolecules 2010, 43 (15), 6245-6260.

(52) Kiskan, B.; Zhang, J.; Wang, X.; Antonietti, M.; Yagci, Y. Mesoporous graphitic carbon nitride as a heterogeneous visible light photoinitiator for radical polymerization. ACS Macro Lett. 2012, 1 (5), 546-549.

(53) Poostforooshan, J.; Badiei, A.; Kolahdouz, M.; Weber, A. P. Synthesis of spherical carbon nitride-based polymer composites by continuous aerosol-photopolymerization with efficient light harvesting. ACS Appl. Mater. Interfaces 2016, 8 (33), 21731-21741.

(54) Sheng, W.; Li, W.; Tan, D.; Zhang, P.; Zhang, E.; Sheremet, E.; Schmidt, B. V. K. J.; Feng, X.; Rodriguez, R. D.; Jordan, R.; Amin, I. Polymer brushes on graphitic carbon nitride for patterning and as SERS active sensing layer via incorporated nanoparticles. ACS Appl. Mater. Interfaces 2020, 12 (8), 9797-9805.

(55) Zhang, L.; Ye, G.; Huo, X.; Xu, S.; Chen, J.; Matyjaszewski, K. Structural Engineering of Graphitic Carbon Nitrides for Enhanced Metal-Free PET-RAFT Polymerizations in Heterogeneous and Homogeneous Systems. ACS Omega 2019, 4 (14), 16247-16255.

(56) Dadashi-Silab, S.; Tasdelen, M. A.; Kiskan, B.; Wang, X.; Antonietti, M.; Yagci, Y. Photochemically mediated atom transfer radical polymerization using polymeric semiconductor mesoporous graphitic carbon nitride. Macromol. Chem. Phys. 2014, 215 (7), 675681.

(57) Fu, Q.; Ruan, Q.; McKenzie, T. G.; Reyhani, A.; Tang, J.; Qiao, G. G. Development of a Robust PET-RAFT Polymerization Using Graphitic Carbon Nitride (g-C3N4). Macromolecules 2017, 50 (19), $7509-7516$.

(58) Sun, J.; Schmidt, B. V. K. J.; Wang, X.; Shalom, M. SelfStanding Carbon Nitride-Based Hydrogels with High Photocatalytic Activity. ACS Appl. Mater. Interfaces 2017, 9 (3), 2029-2034.

(59) Kumru, B.; Shalom, M.; Antonietti, M.; Schmidt, B. V. K. J. Reinforced Hydrogels via Carbon Nitride Initiated Polymerization. Macromolecules 2017, 50 (5), 1862-1869.

(60) Cao, Q.; Heil, T.; Kumru, B.; Antonietti, M.; Schmidt, B. V. K. $\mathrm{J}$. Visible-light induced emulsion photopolymerization with carbon nitride as a stabilizer and photoinitiator. Polym. Chem. 2019, 10 (39), $5315-5323$.

(61) Xu, J.; Antonietti, M. The performance of nanoparticulate graphitic carbon nitride as an amphiphile. J. Am. Chem. Soc. 2017, 139 (17), 6026-6029.

(62) Cao, Q.; Cui, Q.; Yang, Y.; Xu, J.; Han, C.; Li, L. Graphitic carbon nitride as a distinct solid stabilizer for emulsion polymerization. Chem. - Eur. J. 2018, 24 (9), 2286-2291.

(63) Kumru, B.; Molinari, V.; Dünnebacke, R.; Blank, K. G.; Schmidt, B. V. K. J. Extremely Compressible Hydrogel via Incorporation of Modified Graphitic Carbon Nitride. Macromol. Rapid Commun. 2018, 40 (4), 1800712.

(64) Ko, J. W.; Choi, W. S.; Kim, J.; Kuk, S. K.; Lee, S. H.; Park, C. B. Self-Assembled Peptide-Carbon Nitride Hydrogel as a LightResponsive Scaffold Material. Biomacromolecules 2017, 18 (11), 3551-3556.

(65) Cui, Q.; Xu, J.; Wang, X.; Li, L.; Antonietti, M.; Shalom, M. Phenyl-modified carbon nitride quantum dots with distinct photoluminescence behavior. Angew. Chem., Int. Ed. 2016, 55 (11), 36723676.

(66) Barrio, J.; Karjule, N.; Qin, J.; Shalom, M. Condensation of supramolecular assemblies at low temperatures as a tool for the preparation of photoactive $\mathrm{C}_{3} \mathrm{~N}_{3} \mathrm{O}$ materials. ChemCatChem 2019, 11 (24), 6295-6300.

(67) Dolai, S.; Barrio, J.; Peng, G.; Grafmüller, A.; Shalom, M. Tailoring carbon nitride properties and photoactivity by interfacial engineering of hydrogen-bonded frameworks. Nanoscale 2019, 11 (12), 5564-5570.
(68) Kalisman, P.; Nakibli, Y.; Amirav, L. Perfect photon-tohydrogen conversion efficiency. Nano Lett. 2016, 16 (3), 1776-1781.

(69) Wang, Q.; Domen, K. Particulate photocatalysts for light-driven water splitting: mechanisms, challenges, and design strategies. Chem. Rev. 2020, 120 (2), 919-985.

(70) Fu, H.; Pan, C.; Yao, W.; Zhu, Y. Visible-light-induced degradation of rhodamine $\mathrm{B}$ by nanosized $\mathrm{Bi}_{2} \mathrm{WO}_{6}$. J. Phys. Chem. $\mathrm{B}$ 2005, 109 (47), 22432-22439. 